\title{
Indicadores de gestión ambiental y organizacional para establecimientos de alojamiento turístico en Nueva Esparta, Venezuela
}

\author{
Indicators of environmental and \\ organizational management for tourist \\ lodging in Nueva Esparta, Venezuela
}

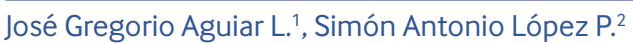

Resumen: La base de esta investigación es la guía básica de autoevaluación en buenas prácticas para la gestión ambiental y organizacional de los establecimientos de alojamiento turístico. Dicha guía es un instrumento de adhesión voluntario que orienta a los establecimientos a asumir la aplicación de un conjunto de medidas técnicas y organizativas que les permitan mejorar su eficiencia en cuanto a producción y prestación del servicio turístico, que deriven en operaciones sostenibles. El objetivo general de esta investigación fue construir indicadores de gestión ambiental y organizacional para establecimientos de alojamientos que propicien la adhesión de las operaciones hoteleras en actividades sostenibles. La metodología utilizada en este estudio fue de diseño no experimental, tipo de campo, nivel descriptivo, transversal y exploratorio. Se seleccionó un establecimiento de alojamiento turístico donde fue aplicada tanto la guía de autoevaluación como el instrumento de indicadores de medición a 122 respondientes. Se obtuvo como resultado un instrumento de indicadores de gestión utilizables en hoteles de distintas categorizaciones. Los resultados obtenidos indicaron una media de 2,71 puntos en la familia de indicadores ambientales, mientras que en las organizaciones se obtuvo una media de 3,59 puntos en la escala de Likert. La principal implicancia de este estudio radica en que el instrumento de indicadores de medición construido puede ser aplicado a cualquier tipo de hotel, independientemente de su categorización. Además, puede aplicarse a cualquier otra empresa de hospitalidad que guarde relación o similitud con las funciones de hospedaje y servicios conexos.

Palabras clave: Gestión ambiental y organizacional, alojamien- to turístico, indicadores ambientales.

Abstract: The basis of this study is the basic guide for self-assessment in good practices for the environmental and organizational management of tourist lodgings. This guide is a voluntary instrument that orients establishments in implementing a set of technical and organizational measures that allows them to improve their efficiency in terms of production and rendering of the tourist service, which they derive from sustainable operations. The general aim of this study was to construct environmental and organizational management indicators for accommodation facilities that support hotel operations in sustainable activities. The methodology used in this study was a non-experimental design, in the field, descriptive, cross-sectional and exploratory. A lodging facility was selected where the self-assessment guide and the instrument of measurement indicators was applied to 122 respondents. The result was an instrument of management indicators that could be used in different types of hotels. The results revealed an average of 2.71 points in the family of environmental indicators, whereas in the organizational indicators an average of 3.59 points was obtained on a Likert scale. The main implication of this study is that the instrument of measurement indicators constructed may be applied to any type of hotel, regardless of its categorization. It can also be applied to any other hospitality company that bears relation to or similarity with the functions of lodging and related services.

Key words: Environmental and organizational management, tourist lodging, environmental indicators.

\section{(Presentado: $1^{\circ}$ de junio de 2017. Aceptado: 19 de octubre de 2017)}

\footnotetext{
${ }^{1}$ Ph.D. en Patrimonio Cultural y Tradición Culinaria. Universidad Simón Bolívar, Venezuela. E-mail: joseaguiarqusb.ve

${ }^{2}$ M.Sc. en Turismo y Recreación, Universidad Simón Bolívar, Venezuela. E-mail: simonlopezqusb.ve
} 


\section{INTRODUCCIÓN}

El Ministerio de Turismo de Venezuela (MINTUR) dispone de un extraordinario instrumento denominado Guía Básica de Autoevaluación en Buenas Prácticas para la Gestión Ambiental y Organizacional de los Establecimientos de Alojamiento Turístico, o simplemente Guía Básica de Autoevaluación en Buenas Prácticas (GBABP). Dicho instrumento no es de carácter mandatorio en su cumplimiento; no obstante, existen leyes y regulaciones que orientan a los prestadores de servicio a adecuarse a las normas sostenibles establecidas globalmente y descritas en la legislación nacional. La guía en cuestión sirvió de base para la creación y construcción de una lista de indicadores de sostenibilidad pertinentes con las operaciones hoteleras. En este sentido, se tomó un establecimiento de alojamiento ubicado en el Estado Nueva Esparta, el cual por razones operativas, de estricta confidencialidad, solicitó al investigador no socializar su nombre, más sí lo que se derive de la investigación. La investigación tuvo como objetivo construir indicadores de gestión ambiental y organizacional para establecimientos de alojamientos que propicien la adhesión de las operaciones hoteleras en actividades sostenibles, a partir de instrumentos de medición de reconocida trayectoria mundial, tales como los dispuestos por la Global Sustainable Tourism Council (GSTC) y la Agenda 21. El presente estudio parte de distintos análisis realizados a diversidad de documentos relacionados con la sostenibilidad, desde el punto de vista ambiental por una parte; y por otra, relacionados con la gestión organizacional. Estos indicadores constituyen un instrumento técnico ambiental y organizativo aplicable a los establecimientos de alojamiento turístico, cuyo objeto será el de mejorar la operación y control de empresas turísticas, esto coadyuvará a la disminución de riesgos laborales, a la vez que proporcionará rentabilidad económica, buen uso de los recursos, control de los costos generales, preservación ambiental y consideración a la comunidad como factor fundamental en la operación hotelera, entre otros. En tal sentido, es necesario asumirlo como una forma de operación en lo sucesivo en el ejercicio del prestador de servicios del establecimiento de alojamiento turístico, su aplicación recurrente y su fiel cumplimiento. En definitiva, la sostenibilidad insta a los establecimientos de alojamiento a adecuar su filosofía de servicio, en términos de comprender las dimensiones que le componen: social, cultural, ambiental, política y económica; pues, en torno a ellas es que se deberá gestionar la empresa de hospitalidad. Los alcances que se derivarán de ella serán inimaginados, satisfactorios y extraordinarios.

Dado lo anterior, el objetivo general de esta investigación fue construir indicadores de gestión ambiental y organizacional para establecimientos de alojamientos que propicien la adhesión de las operaciones hoteleras en actividades sostenibles, a partir de una guía de autoevaluación gestionaria y de instrumentos de medición de reconocida trayectoria mundial. Los objetivos específicos fueron:

- Identificar las dimensiones bajo las cuales se rigen las prácticas sostenibles en establecimientos de alojamiento turístico.

- $\quad$ Establecer lineamientos orientados a las prácticas sostenibles en establecimientos de alojamiento turístico, a partir de sus implicaciones dimensionales.

- Diseñar los lineamientos bajo los cuales debe regirse la operación de establecimientos de alojamiento turístico, desde su práctica sostenible.

- Aplicar los lineamientos establecidos en torno a la operación de establecimientos de alojamiento turístico, a partir de su práctica sostenible.

- $\quad$ Analizar los resultados derivados de las prácticas sostenibles orientados a la operación de establecimientos de alojamiento turístico.

\section{METODOLOGÍA}

La metodología utilizada en el presente estudio parte de la creación de un lineamiento, su aplicación, recolección de los datos, obtención de información relacionada y su análisis descriptivo del resultado. Se seleccionó un establecimiento de alojamiento turístico donde fue aplicado, tanto la GBABP como el instrumento de indicadores de medición a 122 respondientes, tanto gerentes de línea, como trabajadores regulares del staff. El ámbito en cuestión desea no ser divulgado su nombre mercantil, protegiendo así la confidencialidad de la marca y su operación. El diseño de la investigación se refiere a la estrategia que 
adopta el investigador para responder al problema. En tal sentido el diseño en cuestión ha sido considerado como no experimental, ya que "se observan los hechos tal y como se presentan en su contexto real y en un tiempo determinado o no, para ser luego analizados" (Palella y Martins, 2011). Mientras que el tipo de investigación empleada ha sido la del trabajo de campo, definida como "la recolección de datos directamente de la realidad donde ocurren los hechos" (Fernández, 2007). El trabajo de campo, según el Manual de Trabajos de Grado del Fondo Editorial de la Universidad Pedagógica Experimental Libertador (FEDEUPEL, 2010), "es el análisis sistemático de problemas en la realidad, con el propósito bien sea de describirlos, interpretarlos, entender su naturaleza y factores constituyentes...". Más adelante, agregan Palella y Martins (2011), que un hecho investigativo es de nivel descriptivo "cuando se interpretan realidades de un hecho", a la vez que presenta rasgos de transversalidad y símbolos exploratorios. Finalmente, la modalidad a la que se suscribe esta investigación es la de proyecto factible, la cual consiste en "elaborar una propuesta viable destinada a atender las necesidades específicas, determinadas a partir de una base diagnóstica" (Bavaresco, 2001), que en este caso, el producto derivado del presente estudio será un li- neamiento de prácticas sostenibles, desde donde se adecue el mantenimiento de las piscinas residenciales, a partir de implicaciones dimensionales.

La GBABP es un instrumento de adhesión voluntaria que insta a los establecimientos de alojamiento turístico a adecuarse a un comportamiento ante la conservación y preservación ambiental, a la vez que establece parámetros relacionados con el desempeño organizacional. Representa un instrumento de fácil manejo, que aunque se presenta como un texto dicotómico, donde la respuesta positiva equivale a 1 punto y la respuesta negativa equivale a 0 puntos, sirve de asidero operativo. En consecuencia, el presente estudio ha realizado una determinante modificación a esta guía, construyendo indicadores de gestión, apoyados en otros instrumentos utilizados en la actualidad por instancias internacionalmente reconocidas, tales como la Organización Mundial del Turismo (OMT) y el Consejo Global de Sostenibilidad Turística (GSTC).

Como ya se ha dicho, la GBABP parte de las siguientes vertientes para su aplicación, a la vez que presenta los valores máximos a obtener por cada ítem (Tabla 1).

TABLA 1. Guía Básica de Autoevaluación en Buenas Prácticas (GBABP) para la Gestión Ambiental y Organizacional de los Establecimientos de Alojamiento Turístico.

Áreas de gestión y categorías de análisis de la GBABP

\begin{tabular}{lclc}
\hline Gestión ambiental & & Gestión organizacional & Valor \\
\hline Categoría de análisis & $\begin{array}{c}\text { Valor } \\
\text { máximo }\end{array}$ & Categoría de análisis & 6 \\
\hline Manejo del recurso agua & 9 & Políticas y planes & 11 \\
Consumo de energía & 15 & Recursos humanos & 8 \\
Manejo de residuos & 7 & Comunicación y mercadotecnia & 7 \\
Control de la contaminación & 10 & Manejo de productos de alimentos y bebidas & 9 \\
Información y educación ambiental & 7 & Higiene y mantenimiento & 12 \\
Conservación de la flora y fauna & 6 & Seguridad & 11 \\
Respeto a la cultura local & 5 & Responsabilidad social empresarial & 7 \\
& & Satisfacción del turista & 71
\end{tabular}

Fuente: Guía de Autoevaluación para la Gestión Ambiental y Organizacional de MINTUR (2013). 
La GBABP plantea metodológicamente una organización interna que se describe a continuación:

- Gestión ambiental: Señala las acciones relacionadas con la preservación y conservación del ambiente, a partir de la información que el establecimiento posee. Esta gestión abarca las siguientes categorías: Manejo del recurso agua, entre ella las aguas grises, servidas y potables, una vez señaladas, insta a un aprovechamiento de éstas, de acuerdo a su clasificación; consumo de energía, hace referencia a los recursos energéticos utilizados en el establecimiento, en concordancia con el ambiente; manejo de residuos, hace referencia al adecuado destino que se le proporciona a los residuos del establecimiento, respetando y protegiendo a la salud humana y al ambiente; control de la contaminación, son las medidas legales y técnicas relacionadas con la producción de efectos contaminantes, información, parte de la creación de mecanismos de divulgación y socialización de logros y aspectos preventivos; conservación, hace referencia al conjunto de estrategias que contribuyan a la protección y defensa de las especies animales y vegetales que habitan en la comunidad cercana al establecimiento de alojamiento turístico; y cultura local, son las acciones que reconocen y valoran la cultura y estilos de vida asumidos por la comunidad.

- Gestión organizacional: Está relacionada con las acciones propias de la organización, a partir de las siguientes categorías: Políticas y planes, son las directrices bajo las cuales se rige el establecimiento; recursos humanos, es la actividad humana manifiesta en y por el establecimiento; comunicación y marketing, relacionada con actividades profesionales de investigación de mercado, comercialización y planificación, entre otras; manejo de productos y bebidas, son operaciones inherentes a la transformación de materia prima, en producto vendible y posterior servicio; higiene y mantenimiento, son las acciones, técnicas y sistemas establecidos en la ley o en los procedimientos internos que aseguran salud y calidad de los productos y servicios; seguridad, son las acciones orientadas a minimizar y/o evitar peligro, riesgos, daños, entre otros y así, garantizar bienestar a huéspedes, clientes, visitantes y empleados del establecimiento; responsabilidad social, hace referencia a los deberes de la empresa para con la comunidad, en términos de sociedad, cultura, ambiente, legislaciones y economía, satisfacción del turista, hace referencia al nivel de conformidad que manifiesta el huésped al consumir los productos inherentes al establecimiento de alojamiento turístico.

La GBABP fue aplicada a una unidad de alojamiento, tal y como el mismo instrumento lo exige, donde se obtuvo el siguiente resultado para cada dimensión, esto es la dimensión gestión ambiental (Tabla 2) y la dimensión gestión organizacional (Tabla 3), que a la vez sirvió como base para la construcción de los indicadores descritos aquí.

TABLA 2. Situación actual (resultado) de la gestión ambiental.

\begin{tabular}{lllllc}
\hline & \multicolumn{3}{c}{ Trimestre } & Promedio \\
\cline { 2 - 5 } Manejo del recurso agua & 1 & 2 & 3 & 4 & 2,75 \\
Consumo de energía & 0 & 2 & 3 & 6 & 5,50 \\
Manejo de residuos & 1 & 3 & 8 & 10 & 1,00 \\
Control de la contaminación & 0 & 1 & 1 & 2 & 4,00 \\
Información y educación ambiental & 0 & 1 & 7 & 8 & 5,25 \\
Conservación de la flora y fauna & 3 & 4 & 7 & 7 & 4,50 \\
Respeto a la cultura local & 2 & 4 & 6 & 6 & 2,00 \\
\hline Total & 0 & 1 & 3 & 4 & $\mathbf{2 5 , 0 0}$ \\
\hline
\end{tabular}

Fuente: Datos de la investigación. 
TABLA 3. Situación Actual (resultado) de la gestión organizacional.

\begin{tabular}{llcccc}
\hline & \multicolumn{3}{c}{ Trimestre } & \multirow{2}{*}{ Promedio } \\
\hline & 1 & 2 & 3 & 4 & 3,25 \\
\hline Políticas y planes & 0 & 3 & 4 & 60 & 3,25 \\
Recursos humanos & 0 & 1 & 2 & 10 & 4,50 \\
Comunicación y mercadotecnia & 3 & 4 & 5 & 6 & 2,75 \\
Manejo de productos de alimentos y bebidas & 0 & 3 & 5 & 3 & 6,25 \\
Higiene y mantenimiento & 1 & 6 & 9 & 9 & 8,00 \\
Seguridad & 1 & 7 & 12 & 12 & 5,00 \\
Responsabilidad & 3 & 5 & 6 & 6 & 7,00 \\
Satisfacción del turista & 7 & 7 & 7 & 7 & $\mathbf{4 0 , 0 0}$ \\
\hline Total & $\mathbf{1 5}$ & $\mathbf{3 6}$ & $\mathbf{5 0}$ & $\mathbf{5 9}$ & \\
\hline
\end{tabular}

Fuente: Datos de la investigación.

El resultado global de ambas dimensiones durante los trimestres evaluados se muestra en la Figura 1.

FIGURA 1. Situación final de la medición.

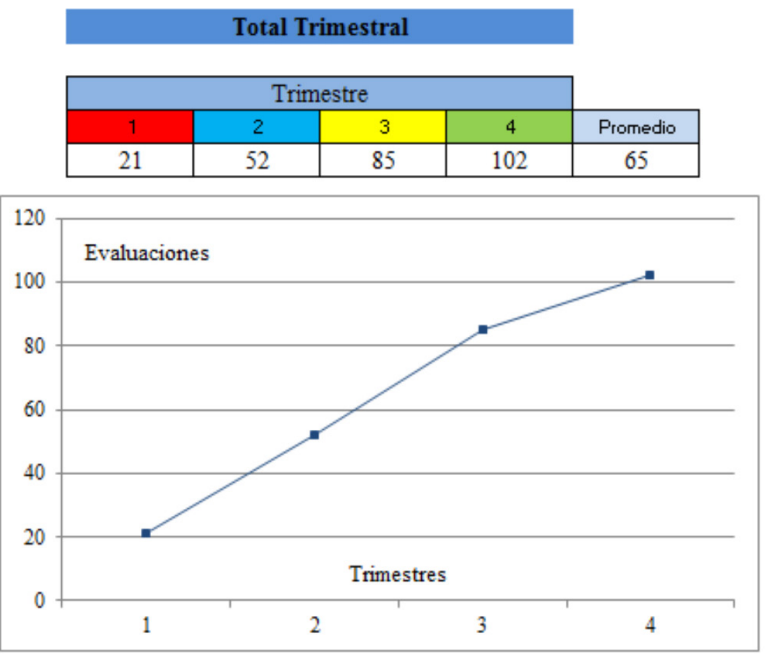

A partir de la escala de valoración de la GBABP se obtiene que el establecimiento de alojamiento evaluado esté clasificado como iniciado (1-32 puntos), comprometido (33-65 puntos), avanzado (66-98 puntos), consolidado (99-130 puntos). Por lo tanto, el establecimiento fue evaluado como comprometido. Es oportuno destacar que el establecimiento de alojamiento seleccionado para la aplicación de esta guía mostró acentuado interés en revisar su gestión, tal y como se evidencia en la Figura 1. El as- cendente mejoramiento hace ver que su gestión podría ser cada vez más sostenible, a medida que se tomen los correctivos que se tengan a bien considerar.

Como ya se dijo, en el presente estudio se aplicaron, tanto la guía de autoevaluación de la gestión, como los indicadores de medición construidos para ambas dimensiones. Seguidamente se detalla el comportamiento de cada uno de los indicadores que constituyen el instrumento aplicado en el citado establecimiento de alojamiento. 


\section{RESULTADOS}

\section{A. Gestión Ambiental}

\section{Manejo del recurso agua potable}

Resulta sorprendente cómo un ítem de tan relevante interés muestre tan bajo promedio, el cual se ubica en un incipiente 1,75 en la escala de Likert. A la vez que se evidencia el desinterés en el ahorro del agua potable, como en el mantenimiento y supervisión de los equipos que la surten. La importancia de la implementación de programas resultan de vital importancia en el aprovechamiento correcto y justo del agua; no obstante, hay una marcada

cultura y desinformación en cuanto a su valor como recurso natural. Su observación el cual registra un 3,25 está orientado a que el agua en el establecimiento de alojamiento seleccionado se obtiene a través de compras de camiones cisternas que llenan los tanques de agua; no obstante, la preocupación radica en el costo que ello genera, más no en su correcta utilización, evidenciando así una desacertada gestión gerencial (Figura 2).

FIGURA 2. Manejo del recurso agua potable.

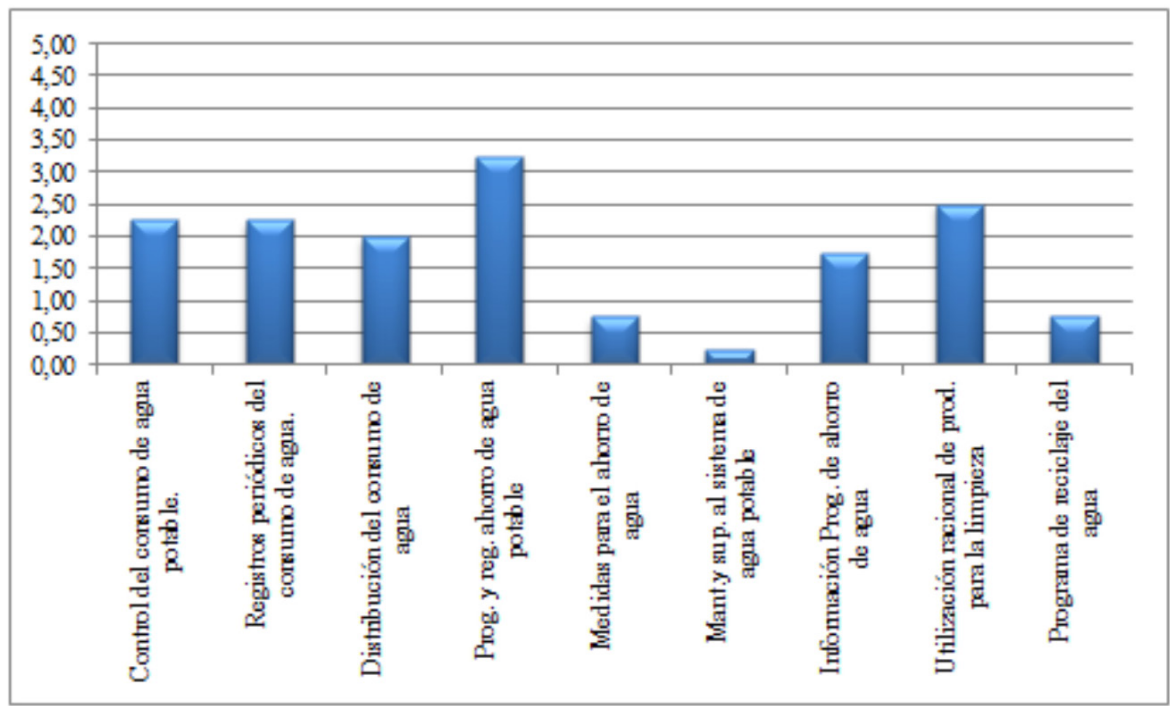

\section{Consumo de energía}

Esta sub dimensión consta de 15 indicadores, los cuales presentaron un promedio de 2,39 en la escala de Likert. Se evidenció durante la aplicación del instrumento que existe un desconocimiento en cuanto a la importancia de recurso energía, su consumo y la utilización de fuentes alternativas. En este sentido se considera a la energía como algo que siempre ha estado allí, no obstante se ignora cuán complejo resulta su obtención. Los controles que se determinaron, en cuanto al consumo, están estrictamente orientados a la disminución de costos en el pago de las facturas mensuales del servicio y no por el correcto y justo aprovechamiento de dicho recurso. El no aprovechamiento de la iluminación natural, indicador que se ubica en 1,85 de la escala utilizada, muestra el marcado desconocimiento en cuanto al tema energético, lo cual exige una urgente intervención de procesos de adiestramiento e información relativos al hecho sostenible, con énfasis en el uso de la energía. Las campañas están orientadas hacia el consumo restringido por parte de los huéspedes, estrategia que persigue un bajo costo en las facturas mensuales del servicio (Figura 3). 
FIGURA 3. Consumo de energía.

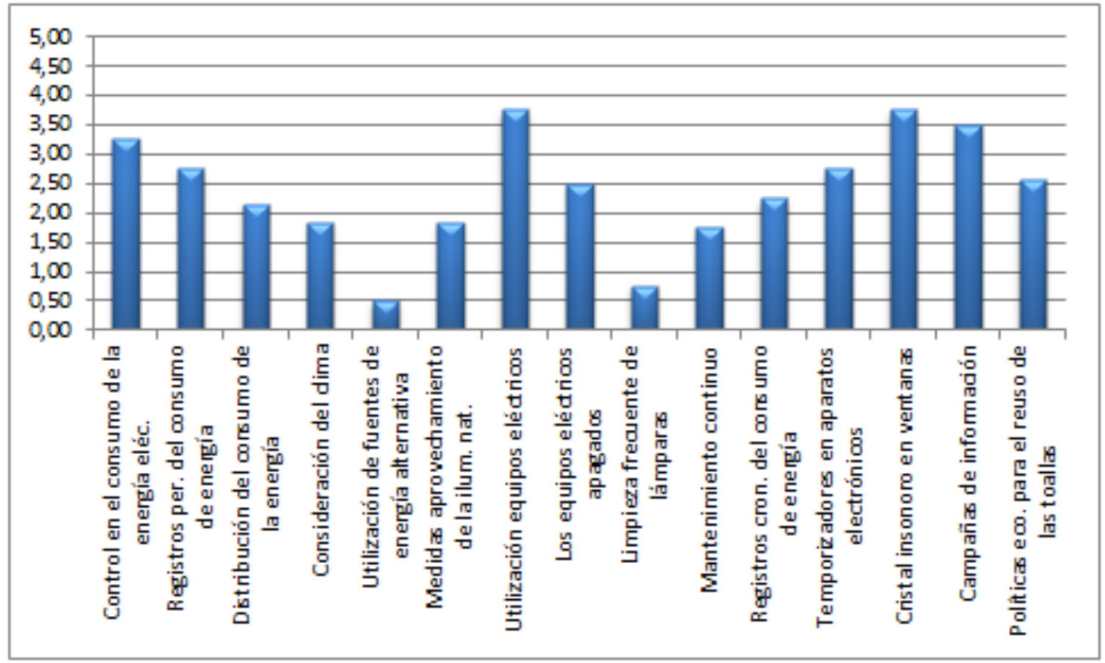

\section{Manejo de residuos}

Esta sub dimensión se ubica en un promedio 1,25 en la escala de Likert, evidenciando que no existe o es casi nulo el registro de las cantidades de residuos generados, a la vez que no se contemplan planes o programes para su gestión. Tampoco existe un aprovechamiento de los desechos, pudiendo ello generar recursos para programas de interés cultural local, si fuera el caso. El seguimiento a esa sub dimensión está orientado hacia el retiro inmediato de éstos, a los fines de no generas malos olores en las adyacencias del establecimiento, más no por reconocer en los desechos una posibilidad de darle un uso productivo ( $\mathrm{Fi}$ gura 4).

FIGURA 4. Manejo de residuos.

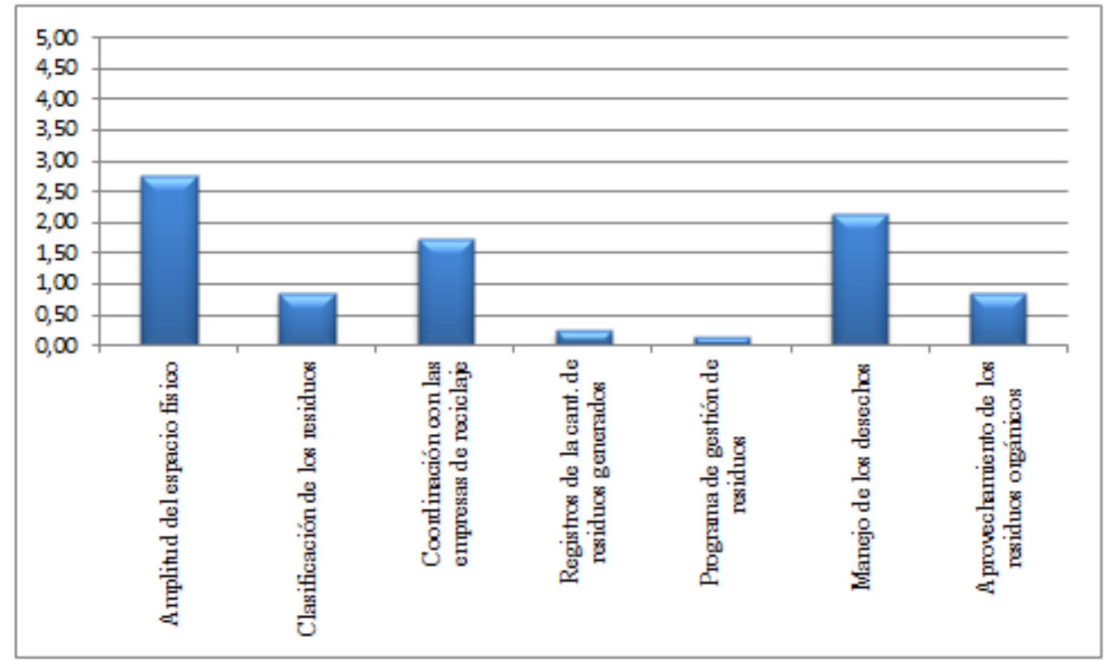




\section{Control de la contaminación}

Ubicado en un promedio 3,01 en la escala de Likert, encontramos esta sub dimensión. Se evidencia su último indicador, ambientes musicales, ubicado en 4,75 se corresponde con la disposición de espacios agradables para el huésped. No obstante, el resto de los indi- cadores no mostraron evaluaciones significantes y favorables. Ello significa descuido con las aguas servidas, con aguas pluviales para su reutilización, tratamiento de las aguas servidas, la basura y su generación de gases (Figura 5).

FIGURA 5. Control de la contaminación.

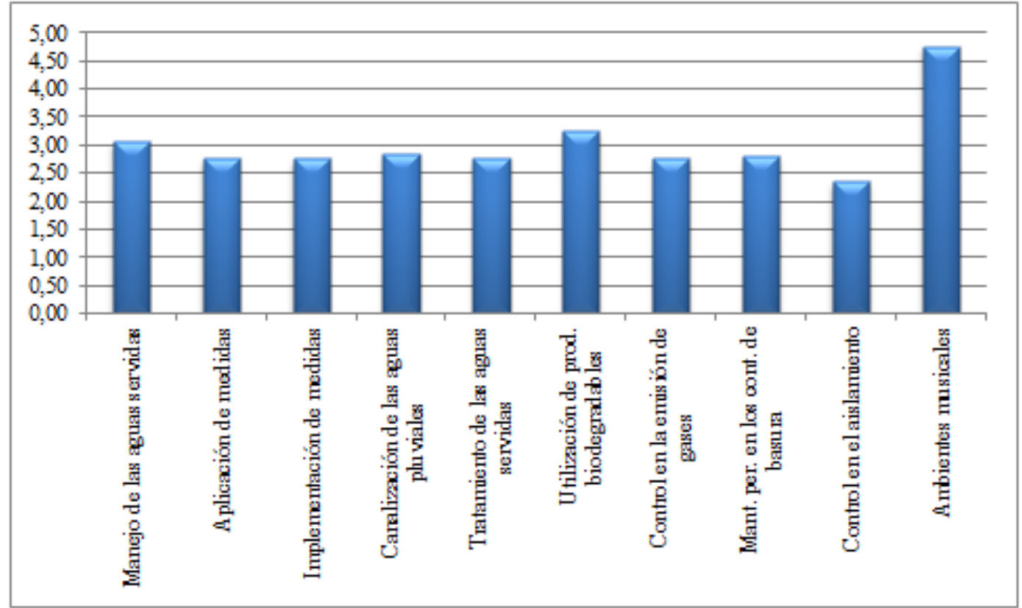

\section{Información y educación ambiental}

Un promedio 4,35 en la escala de Likert obtiene esta sub dimensión, la cual consta de 7 indicadores. En ellos se evidencia, de manera casi unánime, un equilibrio entre los respondientes, en el entendido de que la sensibilidad, respeto y cuido del ambiente y la divulgación de estrategias para su mantenimiento hacen del hotel un espacio agradable, más allá del confort y el lujo que dispone, sino por ejercicio de educación a clientes, huéspedes, visitante y staff en relación con sus espacios naturales y el entorno paisajístico del hotel. Con frecuencia se le deja esta responsabilidad a las instancias de gobierno que regulan la preservación y protección del ambiente. Entonces resulta sumamente gratificante que los hoteles se dediquen a realizar aportes significativos en materia ambiental, en relación con su divulgación y/o su ejercicio educativo (Figura 6).

FIGURA 6. Información y educación ambiental.

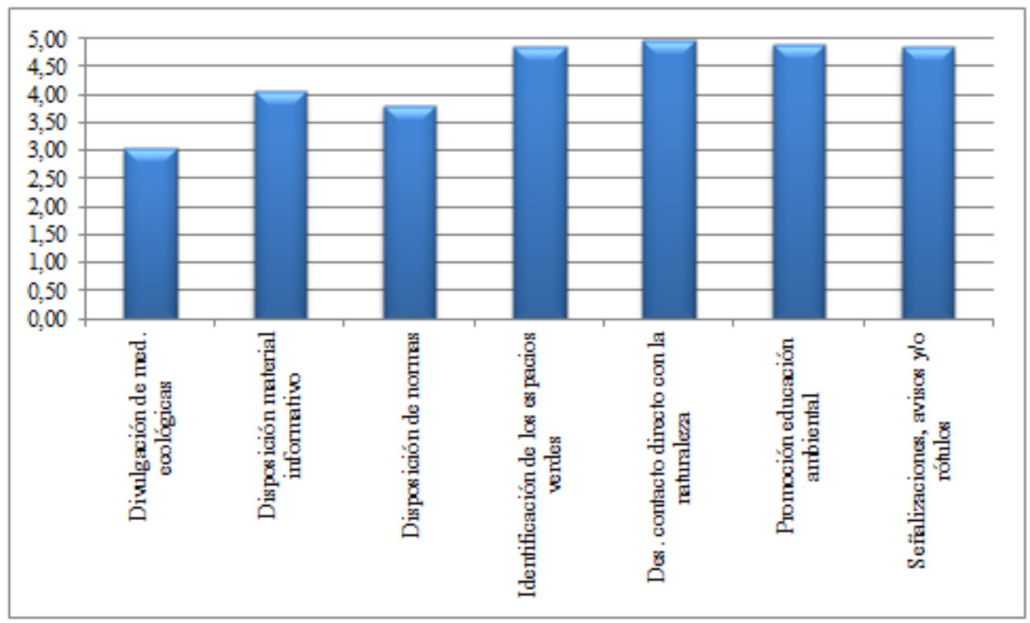




\section{Conservación de la flora y fauna}

Muy pertinente con la sub dimensión anterior está la conservación de la flora y fauna del lugar donde se ubica el hotel estudiado. Aquí se promedian los indicadores en 3,27 puntos en la escala de Likert. Los hoteles en general dedican especial interés en disponer espacios naturales donde árboles, aves y otras especies hagan vida y se complementen con sus espacios. A partir de ello, se proporciona un mantenimiento, seguimiento y cuidado, que por una parte embellece el recinto, mientras que se multiplican espacios naturales en los espacios donde se ubica el hotel seleccionado (Figura 7).

FIGURA 7. Conservación de la flora y fauna.

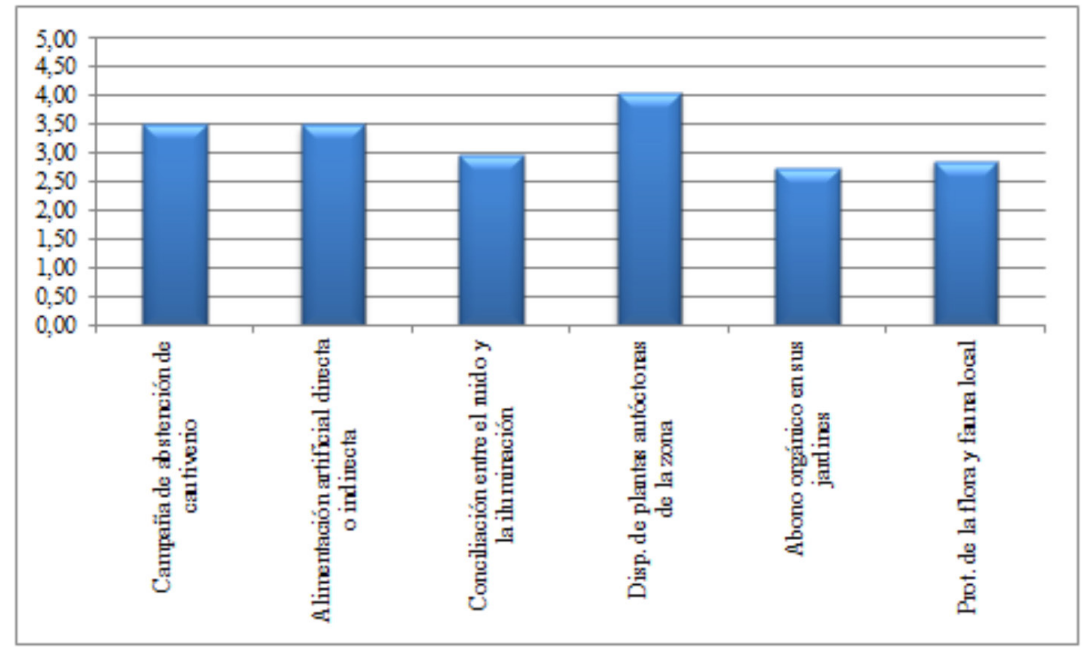

\section{Respeto a la cultura local}

La cultura no es otra cosa que la huella que las sociedades organizadas dejan a su paso por el tiempo. Esta cultura local no puede ser vista desde lejos por las empresas hoteleras que operan en un lugar dado. Es por ello que se ha considerado esta sub dimensión como eje fundamental en el hecho sostenible de estas empre- sas. Ella promedia 2,95 puntos en la escala de Likert. Lo creativo de esta consideración se pudiera contemplar la unión de los cultores de la zona con los empleados y huéspedes del hotel, a los fines de que todos encarnen la materialización cultural regional en los espacios del hotel (Figura 8).

FIGURA 8. Respeto a la cultura local.

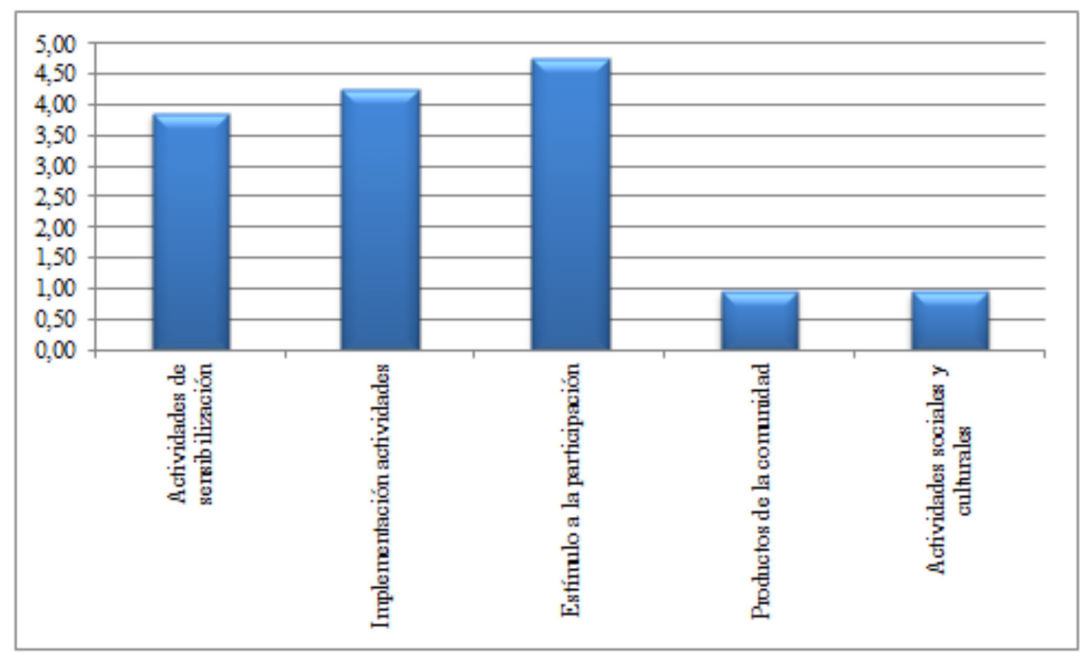


Finalmente, la dimensión ambiental promedia 2,71 puntos en la escala de Likert, siendo una evaluación sumamente baja, una vez analizados todos los indicadores aquí incluidos.

\section{B. Gestión Organizacional}

\section{Políticas y planes}

La sostenibilidad, dentro de los ejercicios operativos de las empresas hoteleras, resulta de sumo interés, no obstante, materializarla no es tarea fácil. Promedia 3,35 puntos en la escala de Likert. Lo trascendente de la sostenibilidad es equilibrar cinco dimensiones para su efectivo alcance, ellas son la social, la cultural, la ambiental, la política y la económica. En esta sub dimensión se dispone un escenario muy favorable en relación con el hecho sostenible, puesto que evidencia un equilibrado promedio. No solo se trata aquí de la implementación de planes, sino de su fiel seguimiento, acompañado de los correctivos de rigor, a la vez que la efectiva socialización a clientes, huéspedes, visitantes y staff hace de esta gesta, una cristalización de la sostenibilidad duradera en el tiempo. La sostenibilidad está asumiendo la vanguardia de toda gestión empresarial, apegándose así a una nueva era en las operaciones en general, pues, es la mejor vía para perdurar con éxito en sus operaciones (Figura 9).

FIGURA 9. Gestión organizacional. Políticas y planes.

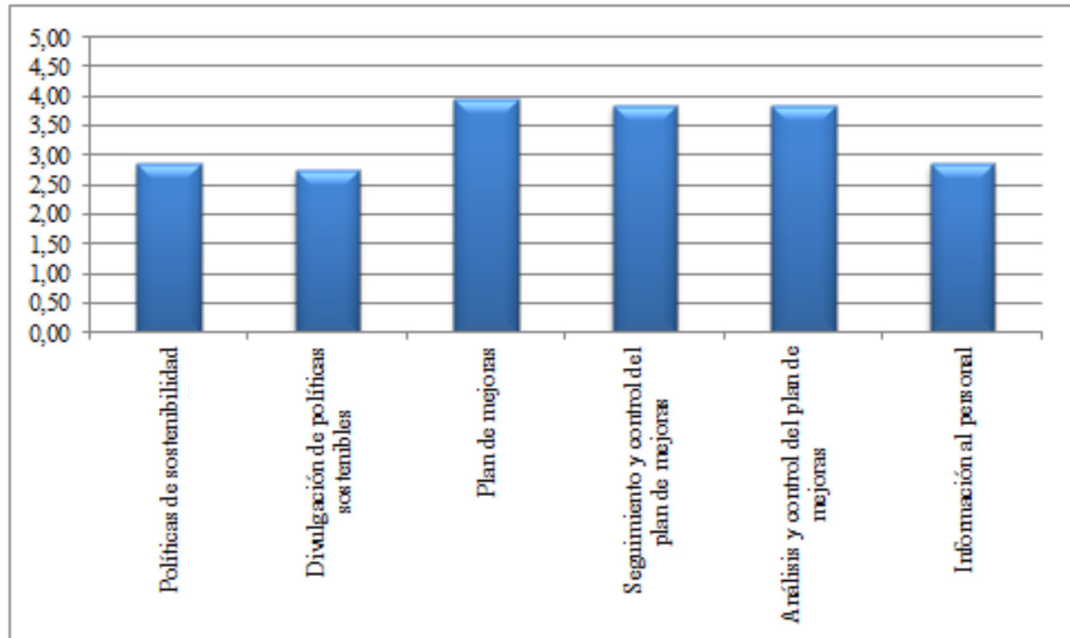

\section{Capital humano}

Se evidencia un promedio 4,08 en la escala de Likert, siendo muy satisfactorio, en cuanto a los resultados de la aplicación del instrumento en el hotel. La redacción de los ítems de esta sub dimensión está dirigida hacia el compromiso de la empresa para con sus empleados y/o asociados. Es común encontrar en las operaciones hoteleras un grupo de trabajadores no vinculados con la marca, que se niegan a incorporarse a la línea empresarial hotelera; no obstante, el hotel mantiene su disposición a abrazar a sus trabajadores y proporcionarles beneficios para todos por igual (Figura 10). 
FIGURA 10. Capital humano.

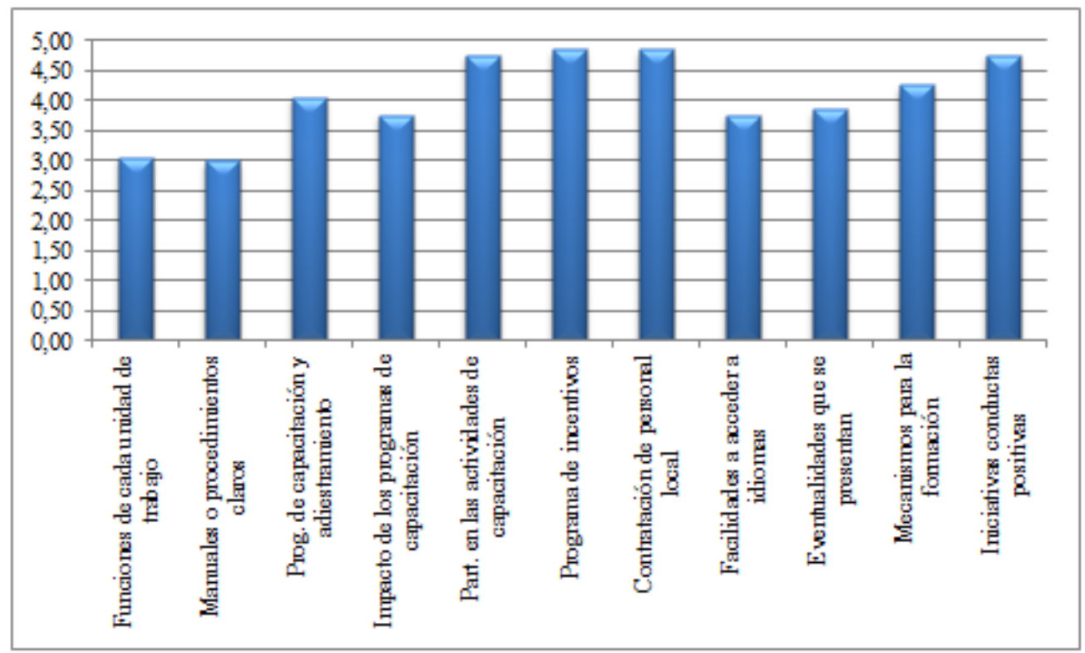

\section{Comunicación y mercadotecnia}

Aquí se tratan temas relacionados a las estrategias y materialización de los alcances de sus planes mercadológicos y sus maneras de divulgación. A tal efecto esta sub dimensión se ubica en un promedio de 3,62 puntos en la escala de Likert. Los planes mercadológicos, evaluados aquí a través de los indicadores señalados, se inclinan al hecho sostenible en la operación hotelera seleccionada para el presente estudio. Lo relevante en estos ítems no es evaluar un plan de mercadeo, pues, toda empresa hotelera debe establecer uno; lo relevante aquí es el vínculo del plan en sí con el hecho sostenible, a la vez que se perfilan sus potencialidades orientadas hacia el mismo punto y la efectiva promoción de su producto (Figura 11).

FIGURA 11. Comunicación y mercadotecnia.

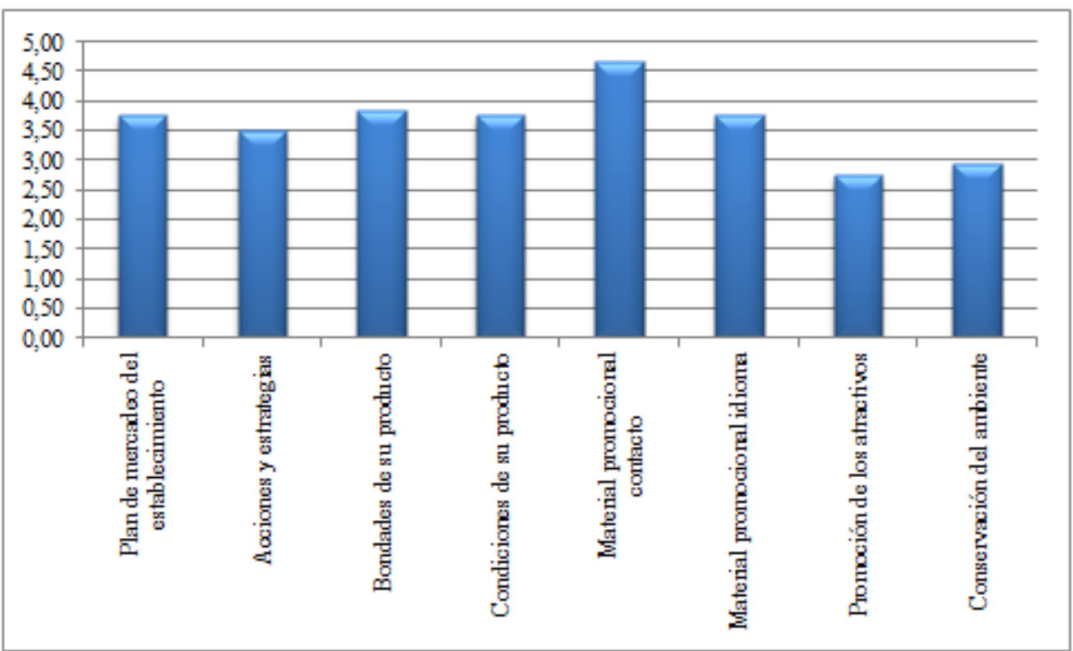




\section{Manejo de productos de A\&B}

La evaluación realizada en relación con el manejo de los alimentos y las bebidas se ubicó en un promedio de 2,69 puntos en la escala de Likert, siendo un resultado extremadamente bajo, puesto que aquí se trata de la ingesta de estos rubros, los cuales poseen lineamientos de higiene de trascendencia internacional, y son visible- mente no son cumplidos. Los ítems que conforman esta dimensión resultan ser sumamente básicos, en términos de normativas de higiene; su cumplimiento se refleja en este promedio, a la vez que los equipos con los que operan, aparentan no estar en las mejores condiciones para su uso (Figura 12).

FIGURA 12. Manejo de productos de A\&B.

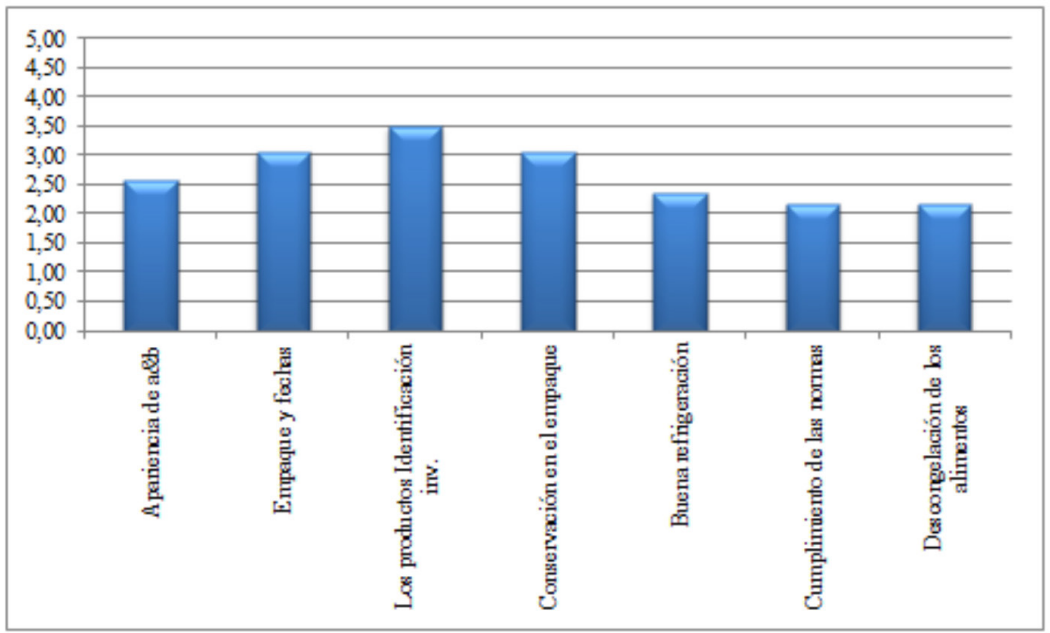

\section{Higiene y mantenimiento}

En esta sub dimensión se ha englobado varios aspectos del mantenimiento y allí se intentó hacerse de la manera más equilibrada; no obstante, el ítem que hace referencia al cuidado de las áreas verdes pudiera estar relacionado con la materialización del cuido de la flora y la fauna o con los procesos y resultados de la edu- cación ambiental, su evaluación favorece significativamente su promedio, el cual se ubica en 3,23 puntos en la escala de Likert. Las estrategias y planes de mantenimiento previstos en el hotel tomado como modelo, apenas se están estructurando mostrando un marcado interés en esta área (Figura 13).

FIGURA 13. Higiene y mantenimiento.

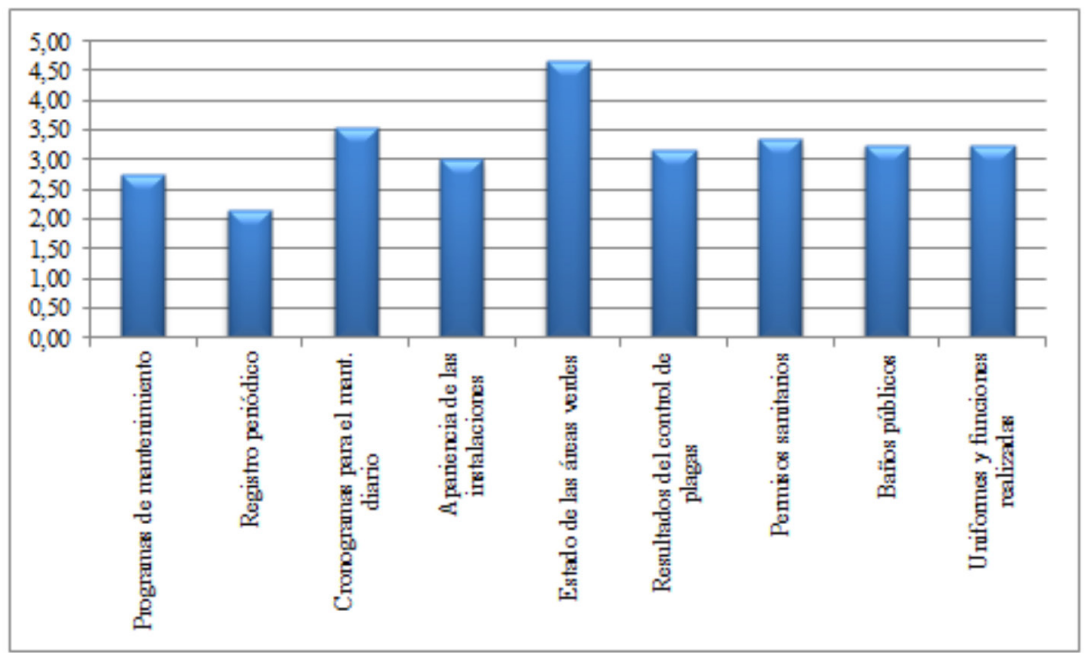




\section{Seguridad}

Esta sub dimensión consta de 12 indicadores, los cuales se ubican en un promedio 3,51 puntos en la escala de Likert. Existe marcado interés de proteger a clientes, huésped y visitantes, dejando al staff poco asistido y evidenciando un moderado interés en su resguardo.
La seguridad debe ser para todos por igual y no únicamente para quien paga una factura, pues, quien labora también requiere, por razones de ley, resguardo y máxima seguridad para el correcto ejercicio de sus funciones (Figura 14).

FIGURA 14. Seguridad.

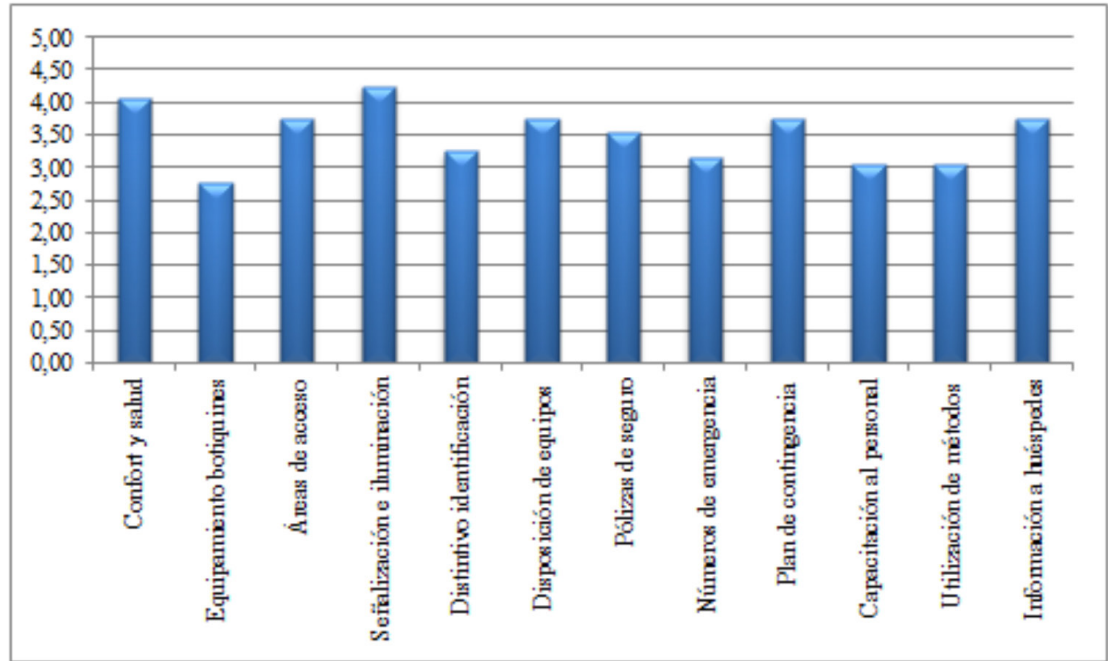

\section{Responsabilidad social y empresarial}

Al igual que la sub dimensión Información y Educación Ambiental, ésta alcanza la mayor evaluación en la aplicación del instrumento, promediando 4,35 puntos en la escala de Likert. Los respondientes proporcionaron una equilibrada evidencia en la gráfica que se muestra, en tal sentido, la empresa hotelera ha mostrado esfuerzos en que las oportunidades de los trabajadores sean pares, a la vez que realiza con frecuencia eventos con trabajadores y otros, menos frecuentes, con la comunidad circundante, estableciendo así, nexos de integración comunitaria (Figura 15).

FIGURA 15. Responsabilidad social y empresarial.

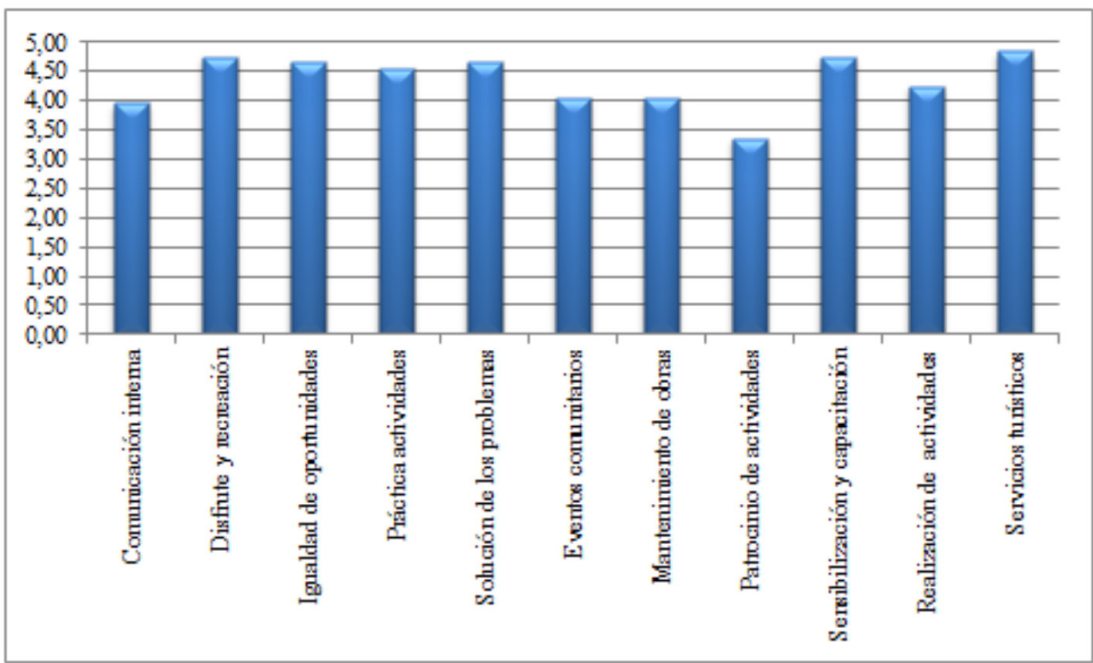




\section{Satisfacción del turista}

El resultado se ubicó en un promedio de 3,92 puntos en la escala de Likert. Este resultado está evaluado como favorable, en cuanto a las gestiones inherentes a proporcionar experiencias trascendentes al cliente, huésped y/o visitante, en términos de consumo del producto hotelero dispuesto. Las expectativas y la satisfacción del huésped están concebidas como el eje fundamental de la operación hotelera tomada como referencia, las que- jas que se pudieran derivar y las compensaciones que pudieran surgir con base a las anteriores, se estrechan en estrategias de control de costos generales. Se evidencia una creciente estrategia de atención al cliente, a la vez que existe un funcionario de control de calidad que comienza sus funciones y está estructurando su gestión, los resultados pudieran evidenciarse más adelante (Figura 16).

FIGURA 16. Satisfacción del turista.

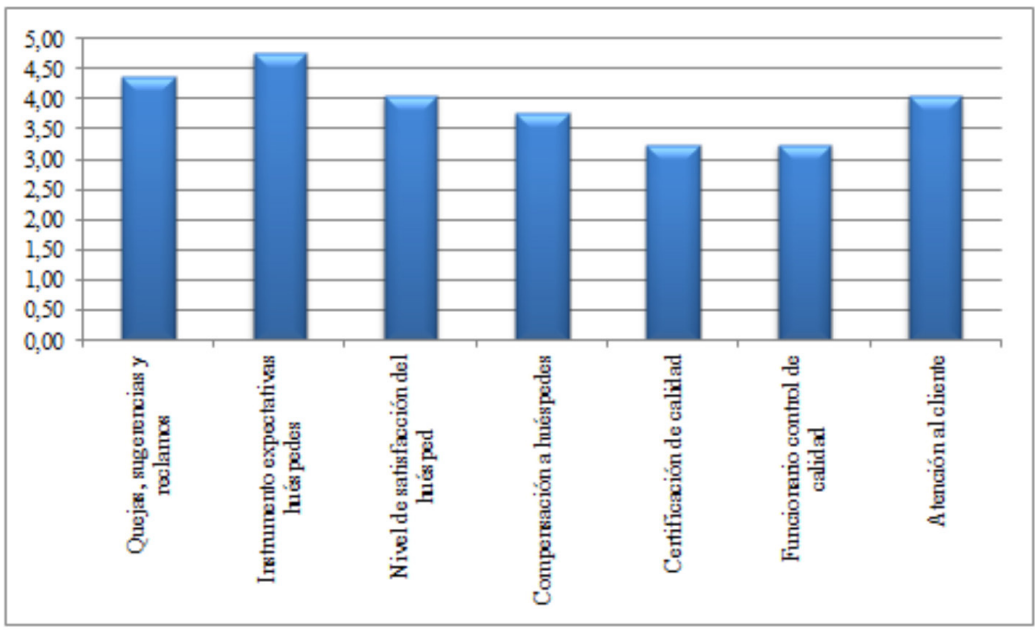

\section{Resumen dimensional}

En la Figura 17 se disponen las dos dimensiones consideradas en la guía de autoevaluación, donde igualmente forman parte de la estructura del instrumento de indicadores de medición construidos aquí. Los promedios obtenidos fueron 2,71 para la gestión ambiental y 3,59 para la gestión organizacional en la escala de Likert. Mientras que el promedio combinado se ubicó en 3,15. Es oportuno señalar que en la dimensión ambiental existe una brecha de 0,88 con respecto a la dimensión organizacional. Esta primera evidencia el notorio desconocimiento e importancia que reviste la preservación y protección, no sólo por razones de ley, sino por el hecho de coadyuvar a vivir en un mundo mejor, para nosotros y para las generaciones por venir. En cuanto a la dimensión organizacional se hace más evidente el interés en hacerlo mejor, pues allí radica la inversión de los empresarios hoteleros. La empresa hotelera, más allá de su estructura organizacional, que está revestida de acierto y notorio éxito, debe hacer un esfuerzo en aprender a interpretar lo que el ambiente pide a gritos y detener su veloz recorrido financiero y dedicar, tanto recursos, como consideración al ambiente, pues este le acoge y le brinda todo su esplendor sin afectar sus finanzas, sino, más bien, incrementándolas de alguna manera. 
FIGURA 17. Resumen dimensional.

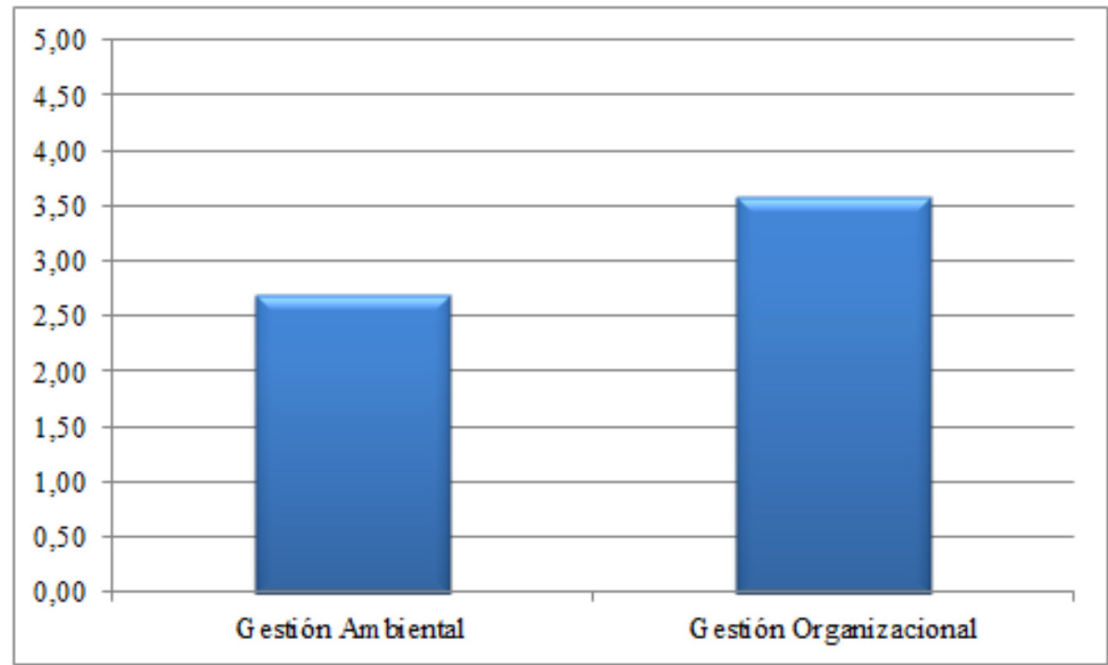

\section{CONCLUSIONES}

En el presente estudio se implementó la Guía Básica de Autoevaluación de Buenas Prácticas, para luego aplicar el instrumento que se construyó. La GBABP sirvió de orientación fundamental. Ello porque la GBSBP arrojó un resultado final de 65 puntos, lo cual ubica al hotel en una clasificación de "comprometido" en la escala de valoración; mientras que por la otra, el instrumento de indicadores de medición construido y aplicado en el hotel referente arrojó un resultado final de 3,15 en la escala de Likert. Si se promedian ambas mediciones, entonces la escala de valoración tendría 16,90 puntos adicionales; no obstante, esta brecha que resulta tiene su origen en que la GBABP tiene una estructura dicotómica, mientras que los indicadores disponen un abanico de respuestas que precisan la realidad sostenible del hotel investigado. Mientras que si se considera el resultado arrojado por la escala de Likert, equivaldría a una evaluación de "avanzado" considerando la misma escala de valoración.
El instrumento de indicadores de medición construido aquí posee como fortaleza que pudiera ser aplicado a cualquier tipo de hotel, independientemente de su categorización, en cuanto a estrellas se refiere, a la vez que pudiera aplicarse a cualquier otra empresa de hospitalidad que guarde relación o similitud con las funciones de hospedaje y servicios conexos.

Los indicadores de gestión ambiental y organizacional construidos aquí sirvieron para dejar una fotografía del hotel tomado como referente, en calidad de indicadores de reporte, a la vez que deja abierta la invitación para que esta empresa replique dicho instrumento, en calidad de monitoreo y logre consolidar su gestión como un hotel sostenible con argumentaciones reales en su operación. De esta manera, podremos propiciar a que uno a uno se vayan sumando hoteles para dar un giro definitivo en sus operaciones, considerando las dimensiones social, cultural, ambiental, política y económica, tal y como se instó en la Agenda 21. 


\section{REFERENCIAS}

Bavaresco, A. (2001). Proceso Metodológico en la Investigación: Cómo hacer un Diseño de Investigación. Editorial Ediluz, Venezuela.

Da Silveira. (2005). Notas sobre el Informe Brundtland o Nuestro Futuro Común.

FEDEUPEL. (2010). Manual de Trabajos de Grado de Especialización y Maestrías y Tesis Doctorales.

Fernández-Guerrero, G. (2010). Metodología de la Investigación. Universidad de Londres.

MINTUR. (2013). Guía de Autoevaluación en Buenas Prácticas para la Gestión Ambiental y Organizacional de
Ios Establecimientos Turísticos.

OMT. (1997). Lo que todo Gestor Turístico debe saber. Guía práctica para el desarrollo y uso de indicadores de turismo sostenible. España.

OMT. (2000). Taller de Indicadores de Sostenibilidad Turística, para los países de Suramérica. Secretaría de Turismo, Argentina.

OMT. (2005). Indicadores de desarrollo sostenible para los destinos turísticos - Guía práctica. Madrid, España.

Palella, S., \& Martins, F. (2011). Metodología de la Investigación Cuantitativa. FEDEUPEL. Venezuela. 